\title{
Placenta accreta
}

\section{Nizami DJ ${ }^{1}$, Awasthi RT ${ }^{2}$, Dash $\mathbf{S}^{1}$, Verghese $\mathbf{J}^{3}$}

${ }^{1}$ Assistant Professor, ${ }^{2}$ Professor, ${ }^{3}$ Tutor, Department of Obstetrics and Gyanaecology, Manipal College of Medical Science, Pokhara, Nepal

\begin{abstract}
Total placenta accreta is a rare condition. Its management is a dilemma. Attempted separation of the placenta in placenta accreta can cause torrential blood loss. Therefore an antenatal diagnosis of placenta accreta permits advance planning of delivery. Two alternatives are caesarean section through the fundus with subsequent immediate hysterectomy, which has traditionally been the treatment of choice or if the patient wishes more children, leaving the placenta in place and managing conservatively ${ }^{1}$. We present a 38 year old lady who was diagnosed to have placenta accreta while performing a caesarean section for a breech presentation. We had to proceed with a total hysterectomy.
\end{abstract}

\begin{abstract}
A 38 year old gravida 5, para 4, living 3 at 39 Aweeks of gestation came to our OPD with a breech presentation. All her previous deliveries were full term normal vaginal deliveries at home with one previous Intra Uterine Foetal Death (IUFD). She did not have any past history of Medical Termination of Pregnancies (MTP's) or uterine surgeries. Serial obstetric examination revealed a large term baby with a breech presentation. Ultrasonography (USG) findings showed a single live foetus of a gestational age of 39 weeks with breech presentation with placenta previa and adequate liquor with an estimated baby weight of 3649 gms. After reviewing the USG report the patient was planned for an elective Lower Segment Caesarean Section (LSCS). LSCS was performed and the baby was delivered by breech extraction. After delivery, it was found that the placenta was covering the cervical Os and could not be delivered by controlled cord traction or manual removal of placenta. No plane of cleavage was found and thus a diagnosis of complete morbid adherent placenta was made. Not much bleeding was encountered as it was a completely adherent placenta. With an on table diagnosis of total placenta accreta a total hysterectomy was performed (Fig.1) .Total blood loss was around $400 \mathrm{ml}$. The removed specimen was sent for histopathological examination (HPE) and was reported as Placenta accreta.
\end{abstract}

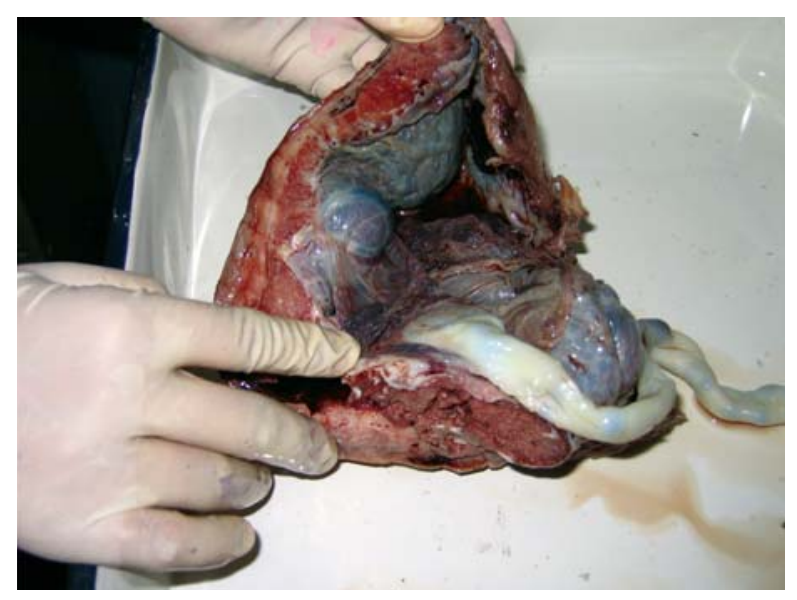

Fig 1: Post op specimen showing the placenta adherent to the uterus

\section{Discussion}

Placenta accreta is a serious obstetric complication where the placenta adheres to the uterine wall because of abnormal development of the decidua basalis. Its incidence varies from 1 in 30000 to 1 per 7000 pregnancies due to the increase in the number of caesarean sections being performed now a day. Its aetiology remains unclear although various risk factors have been mentioned. In the past multiparity was a major risk factor. In 1977, out of 40 cases of pathology proven placenta accreta, there were only 12 women who had had previous caesarean sections.

\footnotetext{
Correspondence

Dr. Deba Jalal Nizami

Assistant Professor, Department of OBG

Kasturba Medical College,

Manipal, Udupi 576104, Karnataka, India

E-mail: mallicks_2000@yahoo.com
} 
However, most women in that series had had three or more children ${ }^{2}$. As the average number of children per patient has decreased, the number of caesarean sections has simultaneously risen so that the latter now appears to be the most important risk factor. Placenta accreta is more frequent in women who have had previous caesarean sections or other types of gynaecologic surgery interrupting the myometrium such as removal of submucous fibroids ${ }^{3,4}$. Miller found that the number of caesarean sections, age, parity and location of the placenta in relation to the scar were all risk factors. Placenta accreta is much more frequent in patients with placenta previa $(880 / 100,000)$ than in upper uterine segment implantations $(5 / 100,000)^{4}$. Other risk factors are a history of a difficult manual removal, multiple abortions with curettage and advanced maternal age. However, $20 \%$ of the cases reviewed by Gielchinsky et al. had no previous risk factors ${ }^{5}$.

USG plays an important role in the antenatal diagnosis of placenta accreta. In early pregnancy the most useful ultrasound finding is implantation of the sac over a uterine scar. Vascular sinuses, appearing as early as 15 weeks, are irregularly shaped, have obvious blood flow when evaluated with colour doppler, and have the highest sensitivity for placenta accreta. Loss of the usual retroplacental clear space as a sole finding will usually be false positive. Magnetic resonance imaging diagnosis is in its infancy and has not yet been proven to add information unless the placenta is posterior. In the future it will hopefully aid in distinguishing placenta accreta from percreta.

Treatment has traditionally been operative; either total or subtotal abdominal hysterectomy, suturing of bleeding sites, or uterine artery ligation after manual removal of the placenta, or curettage with sharp dissection. Riggs et al. have described a different surgical approach where placenta accreta is diagnosed at the time of caesarean section. This involves eversion of the uterus to provide access to the placental site and excision of the placental site followed by closure of the myometrial defect ${ }^{6}$.

Increasingly, conservative treatment has been advocated when blood loss is not excessive and future fertility is desired. In a modern obstetric setting, conservative management is a reasonable alternative when chosen cautiously. Various conservative approaches have been described such as use of Methotrexate, uterine artery embolisation, argon beam coagulation and serial $\beta \mathrm{HCG}$ estimation.

Komulainnen et al. considered that Methotrexate was unlikely to be helpful in cases of placenta accreta because postpartum placental tissue is degenerative and not proliferative ${ }^{7}$.
Similarly, in the case reported by Dunstone et al., there was a steady decline in the $\beta H C G$ level after two doses of Methotrexate and not a rapid decline, as one would expect after Methotrexate. It was suggested in the same report that Methotrexate may be withheld provided there is a rapid spontaneous decline in $\beta \mathrm{HCG}$ levels ${ }^{8}$.

The changes in serum $\beta \mathrm{HCG}$ observed in the study conducted by Matsumara et al. demonstrated that the placenta degenerates spontaneously with a half life of BHCG $5.2+/-0.26$ days, which is longer than that of normal puerperium ${ }^{9} 10$. These findings suggest that the use of Methotrexate might not facilitate degeneration of the placenta at term. Moreover, Methotrexate is an antineoplastic agent, which can cause several side effects, such as myelosuppression and liver dysfunction and it is contraindicated in breast-feeding.

Immediate attempts at traumatic manual removal of the retained placenta at delivery can lead to a situation where hysterectomy becomes inevitable because of bleeding. Therefore, when the woman is stable, it may be prudent to allow spontaneous degeneration and expulsion of the placenta. However, it is difficult to know when to intervene. Matsumara et al. have suggested that the blood loss at the time of removal of retained placenta tended to be less in cases with relatively low serum $\beta \mathrm{HCG}$ levels e.g., $<100 \mathrm{IU}^{8}$.

Thus, serial monitoring of $\beta \mathrm{HCG}$ can be used in the conservative management of women with placenta accreta. Serial monitoring of $\beta \mathrm{HCG}$ can also help decide the timing for safe surgical evacuation.

\section{Conclusion}

Placenta Accreta is a rare condition.USG is helpful in its antenatal diagnosis. Treatment is mainly surgical. However there is a role for conservative treatment in selective cases. Serial B HCG monitoring is helpful in determining the time for safe surgical evacuation of the placenta.

\section{References}

1. Kayem G, Davy C, Goffinet F, Thomas C, Clement D, Cabrol D. Conservative versus extirpative management in cases of placenta accreta. Obstet Gynecol. 2004;104: 531-6.

2. Breen JL, Neubecker R, Gregori C, Franklin J. Placenta accreta, increta, and percreta- A survey of 40 cases. Obstet Gynecol. 1977,49: 43-7.

3. Miller DA, Chollet JA, Goodwin TM. Clinical risk factors for placenta previa-placenta accreta. Am J Obstet Gynecol. 1997; 177:210-14. 
4. Makhseed M, Moussa MA. Placenta accreta in Kuwait: does a discrepancy exist between fundal and praevia accreta? Eur J Obstet Gynecol Reprod Biol. 1999;86: 159-63

5. Gielehinsky Y, Rojanksy N, Fasouliotis S, Ezra Y. Placenta accreta - summary of 10 years: a survey of 310 cases. Placenta. 2002, 23:210-4.

6. Ris J, Jahshan A, Schiavello H. Alternative conservative management of placenta accreta. Journal of Reproductive Medicine. 2000;45:595-7.

7. Komulainen $M$, Väyrynen $M$, Kauko $M$, Saarikoski S. Two cases of placenta accreta managed conservatively. European Journal of Obstetrics \& Gynecology and Reproductive Biology. 1995;62:135-7.
8. Dunstone S, Leibowitz C. Conservative management of placenta praevia with a high risk of placenta accreta. Aust NZ J Obstet Gynaecol. 1998;38: 429-33.

9. Matsumara N, Inoue T, Fakuoka M, Sagawa N, Fujii S. Changes in serum levels of Human Chorionic Gonadotrophin and the pulsatality index of uterine arteries during conservative management of retained adherent placenta. J Obstet Gynaecol Res. 2000;26: 81-7.

10. Reyes F, Winter J, Faiman C. Postpartum disappearance of chorionic gonadotropin from the maternal and neonatal circulations. Am J Obstet Gynecol. 1985;153:486-9.

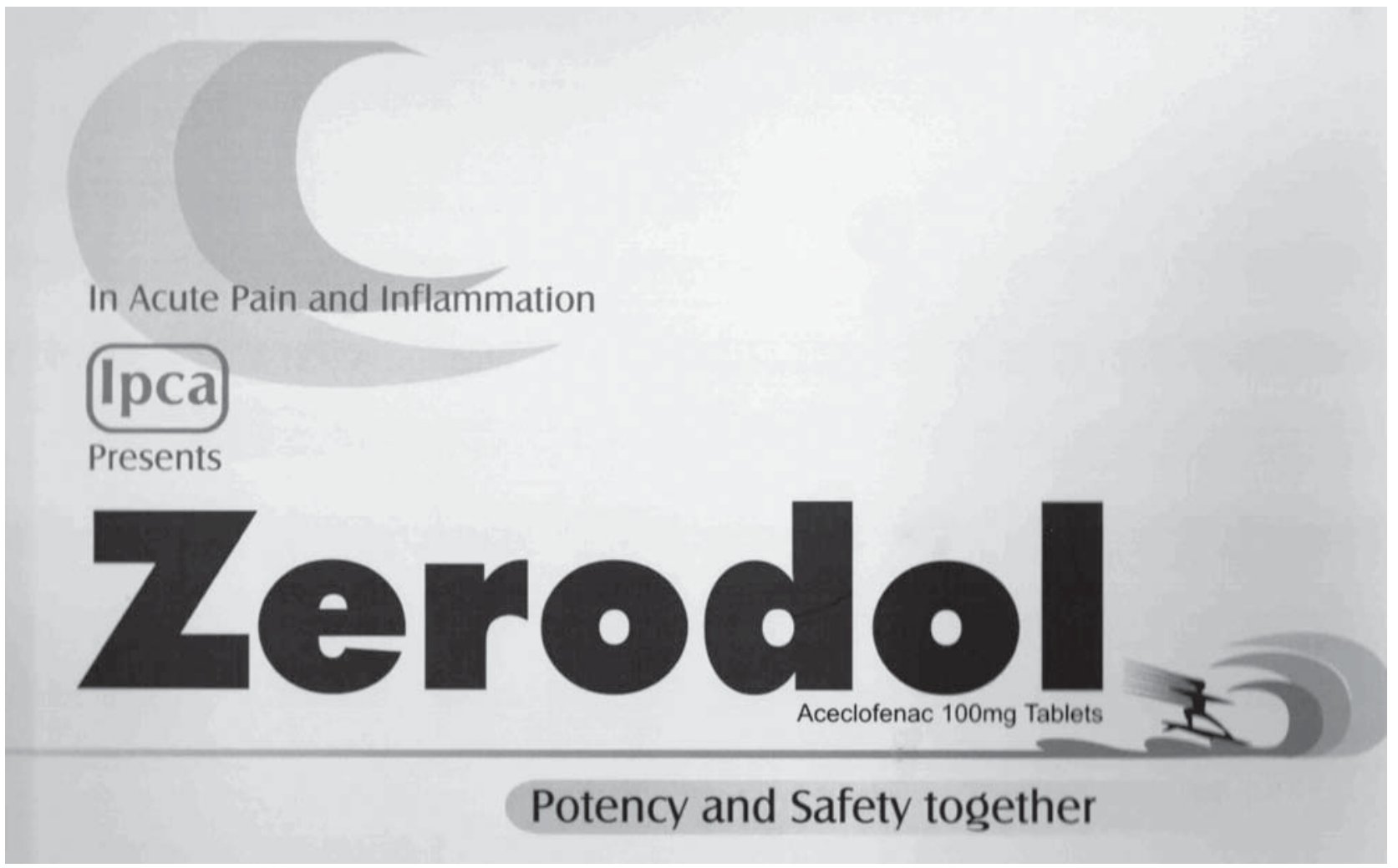

